\title{
THE SYMMETRIC CROSSCAP NUMBER OF A GROUP
}

\author{
COY L. MAY \\ Department of Mathematics, Towson University, Baltimore, Maryland 21252, USA \\ e-mail:cmay@towson.edu
}

(Received 28 December, 1999)

\begin{abstract}
Let $G$ be a finite group. The symmetric crosscap number $\tilde{\sigma}(G)$ is the minimum topological genus of any compact non-orientable surface (with empty boundary) on which $G$ acts effectively. We first survey some of the basic facts about the symmetric crosscap number; this includes relationships between this parameter and others. We obtain formulas for the symmetric crosscap number for three families of groups, the dicyclic groups, the abelian groups with most factors in the canonical form isomorphic to $Z_{2}$, and the hamiltonian groups with no odd order part. We also determine $\tilde{\sigma}(G)$ for each group $G$ with order less than 16 . The groups with symmetric crosscap numbers 1 and 2 have been classified. We show here that there are no groups with $\tilde{\sigma}=3$; this affirms a conjecture of Tucker.
\end{abstract}

2000 Mathematics Subject Classification. Primary 57M60; secondary 20H10, $30 \mathrm{~F} 50$.

1. Introduction. In connection with group actions on compact surfaces, there are several natural parameters associated with each finite group. Here we consider one of the newer ones, the symmetric crosscap number. A finite group $G$ can be represented as a group of automorphisms of a compact non-orientable surface (with empty boundary); that is, $G$ acts on a non-orientable surface. The symmetric crosscap number $\tilde{\sigma}(G)$ is the minimum topological genus of any non-orientable surface on which $G$ acts. Two important related parameters are the symmetric genus and the strong symmetric genus.

We begin by briefly surveying some of the basic facts about the symmetric crosscap number. These facts include relationships between this parameter and others. We also consider upper and lower bounds for the symmetric crosscap number of a group in terms of its order. We examine large groups of automorphisms and the importance of the extended triangle groups; here we also give the connection with regular maps on non-orientable surfaces.

We obtain formulas for the symmetric crosscap number for three families of groups, the dicyclic groups, the abelian groups with most of the factors in the canonical form isomorphic to $Z_{2}$, and the hamiltonian groups with no odd order part. We also determine $\tilde{\sigma}(G)$ for each group $G$ with order less than 16 .

A natural problem to classify, for each value of the integer $\tilde{\sigma}$, the groups of symmetric crosscap number $\tilde{\sigma}$. This has been done for the groups with symmetric crosscap numbers 1 and 2 [19, Theorems 3.2 and 4.1]. The groups with $\tilde{\sigma}=1$ act on the projective plane, and those with $\tilde{\sigma}=2$ act on the Klein bottle. We show that there are no groups with $\tilde{\sigma}=3$. This resolves a conjecture of Tucker [19, p. 1118]. 
2. Preliminaries. We shall assume that all surfaces are compact and without boundary. If $S$ is a surface, then $S$ is characterized topologically by orientability and its topological genus $p$.

The Euler characteristic $\chi(S)$ of an orientable surface $S$ of topological genus $p$ is $\chi(S)=2-2 p$. If the surface $S$ is non-orientable with $p$ crosscaps (or topological genus $p$ ), then the Euler characteristic is $\chi(S)=2-p$.

Regardless of orientability, the surface $S$ can carry a dianalytic structure [1] and be considered a Klein surface or a non-singular algebraic curve over $\mathbf{R}$. Thus the surface $S$ also has an algebraic genus $g$. If $S$ is orientable, then $S$ is a classical Riemann surface, and the algebraic genus and the topological genus agree. If $S$ is a nonorientable Klein surface, then the algebraic genus $g=p-1$, where $p$ is the crosscap number. It is sometimes convenient to use the algebraic genus.

Non-euclidean crystallographic (NEC) groups are a standard tool for studying group actions on surfaces. Associated with each NEC group $\Gamma$ is its signature, which has the form

$$
\left(p ; \pm ;\left[\lambda_{1}, \ldots, \lambda_{\mathrm{r}}\right] ;\left\{\left(v_{11}, \ldots, v_{1 \mathrm{~s}_{1}}\right), \ldots,\left(v_{\mathrm{k} 1}, \ldots, v_{\mathrm{ks}_{\mathrm{k}}}\right)\right\}\right)
$$

The quotient space $U / \Gamma$ is a surface with topological genus $p$ and $k$ holes. The surface is orientable if the plus sign is used and non-orientable otherwise. Associated with the signature (2.1) is a presentation for the NEC group $\Gamma$. Further, the noneuclidean area $\mu(\Gamma)$ of a fundamental region for $\Gamma$ can be calculated directly from its signature [15, p. 235]. An excellent reference for the basics about NEC groups is the monograph [2].

Let $X$ be a Klein surface of algebraic genus $g \geq 2$. Then $X$ can be represented as $U / K$, where $K$ is a surface group. Let $G$ be a group of dianalytic automorphisms of the Klein surface $X$. Then there are an NEC group $\Gamma$ and a homomorphism $\phi: \Gamma \rightarrow G$ onto $G$ such that kernel $\phi=K$. If $X$ is a Riemann surface, then $K$ is a Fuchsian surface group and

$$
\mu(K)=4 \pi(\mathrm{g}-1)
$$

If $X$ is a non-orientable Klein surface, then the surface group $K$ is a proper NEC group and

$$
\mu(K)=2 \pi(\mathrm{g}-1)
$$

Let $G$ be a finite group and let $\theta: \Gamma \rightarrow G$ be a homomorphism of the proper NEC group $\Gamma$ onto $G$ that preserves the orders of the elements of finite order in $\Gamma$. Then the kernel $K$ of $\theta$ is a surface group, and $G$ acts on the compact surface $U / K$. There is a criterion for the orientability of $U / K$.

Proposition A [14]. The surface $U / K$ is non-orientable if and only if $\theta\left(\Gamma^{+}\right)=G$. Equivalently, the surface $U / K$ is orientable if and only if $\theta\left(\Gamma^{+}\right)$has index 2 in $G$.

Now the genus of the surface $U / K$ on which the group $G \cong \Gamma / K$ acts can be calculated from $[\Gamma: K]$ and either (2.2) or (2.3). In particular, if $U / K$ is a nonorientable surface with $c$ crosscaps, then $g=c-1$ and

$$
c=2+|G| \cdot \mu(\Gamma) / 2 \pi \text {. }
$$


For more on this general approach, see [14] and [10].

3. Actions on non-orientable surfaces. We first show that each finite group acts on a non-orientable Klein surface. This result is surely something of a folk theorem, and we give a quick proof. The corresponding result for Riemann surfaces is a classical result of Greenburg [6, p. 572].

TheOREm A. Let $G$ be a finite group. Then there is a non-orientable Klein surface $X$ such that $G$ is a group of automorphisms of $X$.

Proof. If the group $G$ is cyclic or dihedral, then $G$ acts on the projective plane $\left[19\right.$, p. 1111]. Suppose that $G$ has generators $z_{1}, \ldots, z_{r}$, where $r \geq 3, o\left(z_{i}\right)=m_{i}$ and the product $z_{1} z_{2} \cdots z_{r}=1$. Let $\Gamma$ be the NEC group with signature $\left(1 ;-;\left[m_{1}, \cdots, m_{r}\right] ;\{\}\right)$. The group $\Gamma$ is generated by $x_{1}, \ldots, x_{r}, a$ with defining relations

$$
\left(x_{i}\right)^{m_{i}}=x_{1} x_{2} \cdots x_{r} a^{2}=1 .
$$

Define a homomorphism $\phi: \Gamma \rightarrow G$ by $\phi\left(x_{i}\right)=z_{i}, \phi(a)=1$, and let $K=\operatorname{ker} \phi$. Then $K$ contains no elements of finite order, and obviously $\phi\left(\Gamma^{+}\right)=G$, since each $x_{i}$ is in $\Gamma^{+}$. Thus $K$ is a non-orientable surface group, and $G \cong \Gamma / K$ is a group of automorphisms of the non-orientable Klein surface $X=U / K$.

Thus each finite group $G$ acts on a non-orientable surface (as a group of homeomorphisms), and it is natural to seek the surface of smallest topological genus. The symmetric crosscap number $\tilde{\sigma}(G)$ is the minimum crosscap number (or topological genus) of any non-orientable surface on which $G$ acts [12]. This parameter $\tilde{\sigma}(G)$ could also be called the symmetric non-orientable genus of $G$ [19]. The symmetric crosscap number, in one guise or another, has been studied by several mathematicians working on Klein surfaces; for example, see [3] and [7] as well as [19].

An immediate consequence of (2.4) and our proof of Theorem A is an upper bound for $\tilde{\sigma}(G)$.

COROLlARY 1. Let $G$ be a finite group with generators $z_{1}, \cdots, z_{r}$, where $o\left(z_{i}\right)=m_{i}$ and the product $z_{1} z_{2} \cdots z_{r}=1$. Then

$$
\sigma(G) \leq 2+|G|\left[r-1-\sum_{i=1}^{r} \frac{1}{m_{i}}\right] .
$$

While this upper bound is general and perhaps of a little theoretical interest, the bound is not very good, since rank $\Gamma=r$ but rank $G \leq r-1(a$ is in kernel $\phi)$. It is possible to get an improved, useful upper bound by assuming that $G$ has an involution in its center.

THEOREM 1. Let $G$ be a finite group with generators $z_{1}, \cdots, z_{r}$, where $o\left(z_{i}\right)=m_{i}$. If there is an involution $w$ in the center of $G$, then

$$
\sigma(G) \leq 2+|G|\left[r-1-\sum_{i=1}^{r} \frac{1}{m_{i}}\right] .
$$


Proof. In the simple cases $\left(r=1\right.$ and $\left.r=2=m_{1}=m_{2}\right), \tilde{\sigma}(G)=1$ and the inequality holds. Now consider the remaining cases. Let $\Gamma$ be the proper NEC group with signature $\left(0 ;+;\left[m_{1}, \cdots, m_{r}\right] ;\{()\}\right)$. The group $\Gamma$ is generated by $x_{1}, \cdots, x_{r}, e$ and the reflection $c$ with defining relations

$$
\left(x_{i}\right)^{m_{i}}=c^{2}=e c e^{-1} c=x_{1} x_{2} \ldots x_{r} e=1 .
$$

Define a homomorphism $\phi: \Gamma \rightarrow G$ by $\phi\left(x_{i}\right)=z_{i}, \phi(e)=\left(z_{1} z_{2} \ldots z_{r}\right)^{-1}, \phi(c)=w$. Then let $K=$ kernel $\phi$. Now $K$ contains no elements of finite order, and again $\phi\left(\Gamma^{+}\right)=G$, as each $x_{i} \in \Gamma^{+}$. Hence $K$ is a non-orientable surface group, and $G$ acts on the non-orientable surface $X=U / K$. Let $X$ have crosscap number $p$. Then we calculate $p$ from $\mu(\Gamma)$ and (2.4). We see that $\tilde{\sigma}(G) \leq p$. This gives the upper bound.

This improves Corollary 1, since the generators are not required to satisfy $z_{1} z_{2} \ldots z_{r}=1$. Having an involution in the center is a rather special condition, of course. We shall see an application in Section 6.

4. Basic relationships among the parameters and two lower bounds. There are two parameters that are closely related to the symmetric crosscap number. The symmetric genus $\sigma(G)$ of the group $G$ is the minimum genus of any Riemann surface on which $G$ acts (possibly reversing orientation). The strong symmetric genus $\sigma^{0}(G)$ is the minimum genus of any Riemann surface on which $G$ acts preserving orientation. Obviously $\sigma(G) \leq \sigma^{0}(G)$.

Doubling is a standard way to deal with non-orientable surfaces. Associated with each non-orientable surface $X$ is its complex double $X_{c}$ [1, pp. 37-41], a Riemann surface with the same algebraic genus as $X$ and Euler characteristic $\chi\left(X_{c}\right)=2 \chi(X)$. The surface $X_{c}$ has an orientation-reversing involution $\sigma: X_{c} \rightarrow X_{c}$ such that $X_{c} / \sigma=X$. Let $H$ and $G$ denote the automorphism groups of $X$ and $X_{c}$, respectively. Also let $G^{+}$be the subgroup of $G$ consisting of the orientation-preserving automorphisms of $X_{c}$, and set $L=<\sigma>$. Then

$$
H \cong\left\{f \in G^{+} \mid f \sigma=\sigma f\right\}
$$

and thus $G$ contains a subgroup isomorphic to $L \times H \cong Z_{2} \times H$. Here see [1, p. 79] and $[18, \S 6]$.

Now represent the non-orientable surface $X$ as $U / K$, where $K$ is a surface group. We obtain a proper NEC group $\Gamma$ and a homomorphism $\phi: \Gamma \rightarrow H$ onto $H$ such that kernel $\phi=K$ and also $\phi\left(\Gamma^{+}\right)=H$. The surface group $K$ has a canonical Fuchsian subgroup $K^{+}$, and the surface $U / K^{+}$is the complex double of $X$. Further, we have $\Gamma^{+} / K^{+} \cong \Gamma / K \cong H, \quad K / K^{+} \cong<\sigma>$, and $\Gamma / K^{+} \cong<\sigma>\times H$. For more details of this, see $[\mathbf{1 4}, \S 2]$.

It is easy to establish two basic inequalities involving $\tilde{\sigma}$ and other parameters; these inequalities were also pointed out in [12]. Suppose that $G$ is a finite group with symmetric crosscap number $\tilde{\sigma}=\tilde{\sigma}(G)$. Then $G$ acts on a non-orientable Klein surface $X$ with $\tilde{\sigma}$ crosscaps and Euler characteristic $\chi(X)=2-\tilde{\sigma}$. The complex double $X_{c}$ has Euler characteristic $\chi\left(X_{c}\right)=2(2-\tilde{\sigma})$. The genus of $X_{c}$ is $1-\chi\left(X_{c}\right) / 2=\tilde{\sigma}-1$. Now $G$ acts on $X_{c}$ preserving orientation, and $Z_{2} \times G$ acts on $X_{c}$ as well. Therefore 


$$
\begin{gathered}
\tilde{\sigma}(G) \geq \sigma^{0}(G)+1, \\
\tilde{\sigma}(G) \geq \sigma\left(Z_{2} \times G\right)+1 .
\end{gathered}
$$

5. Large groups and regular maps. Large groups of automorphisms of surfaces are quotients of triangle groups. A triangle group is a Fuchsian group with signature $(0 ;+;[\ell, m, n] ;\{\})$, where $1 / \ell+1 / m+1 / n<1$. We denote a group with this signature by $\Gamma(\ell, m, n)$. An extended triangle group is an NEC group with signature $(0 ;+;[] ;\{(\ell, m, n)\})$; we denote this group by $\Gamma[\ell, m, n]$. The basic result about triangle groups and large groups of orientation-preserving automorphisms of Riemann surfaces is given in $[\mathbf{1 1}, \S 2]$ and $[\mathbf{1 0}, \S 4]$; also see [16, p. 22] and [18, Theorem 12].

We give the corresponding result for non-orientable surfaces. Let $\Gamma=\Gamma(k, m, n)$ be a triangle group. In general, there are two types of proper NEC groups with canonical Fuchsian subgroup isomorphic to $\Gamma[\mathbf{1 6}$, p. 21]. One is the extended triangle group $\Gamma[k, m, n]$. The other type only occurs when $\Gamma=\Gamma(m, m, n)$ has two equal periods. This group has signature $(0 ;+;[m] ;\{(n)\})$ and presentation

$$
c^{2}=x^{m}=\left(c x c x^{-1}\right)^{n}=1
$$

We denote a group with this signature by $\Delta(m, n)$.

Lemma 1. Let $G$ be a group of automorphisms of a non-orientable Klein surface $X$ with crosscap number $p \geq 3$. If $|G|>24(p-2)$, then $G$ is a quotient of an extended triangle group $\Gamma[2, m, n]$ with $m \neq n$. If $24(p-2) \geq|G|>12(p-2)$, then $G$ is a quotient of an extended triangle group $\Gamma[2, m, n]$ with $m \neq n$, except in the following cases; in each case $G$ is a quotient of the proper NEC group listed.
(1) $|G|=24(p-2), \Gamma[3,3,4]$,
(2) $|G|=24(p-2), \Delta(3,4)$,
(3) $|G|=20(p-2), \Gamma[2,5,5]$,
(4) $|G|=20(p-2), \Delta(5,2)$,
(5) $|G|=15(p-2), \Gamma[3,3,5]$,
(6) $|G|=15(p-2), \Delta(3,5)$.

Proof. Represent $X$ as $U / K$, where $K$ is a non-orientable surface group, and obtain a proper NEC group $\Lambda$ and a homomorphism $\alpha: \Lambda \rightarrow G$ onto $G$ such that kernel $\alpha=K$. Further $\alpha\left(\Lambda^{+}\right)=G$ and $U / K^{+}$is the complex double $X_{c}$, a Riemann surface with genus $g=p-1$. Then $G \cong \Lambda^{+} / K^{+}$acts on $X_{c}$ preserving orientation. It is now a routine matter of applying Lemma $\mathrm{A}$ of $\left[\mathbf{1 0}, \mathrm{p}\right.$. 117] to $\Lambda^{+}$and considering the possibilities for $\Lambda$.

The extended triangle group producing the largest possible order is $\Gamma[2,3,7]$. If the group $G$ acts on a non-orientable Klein surface with crosscap number $p \geq 3$, then $|G|$ is at most $84(p-2)=84(g-1)$. Since $G$ acts on the complex double $X_{c}$ preserving orientation, this also follows from the classic bound of Hurwitz. See [14]. One consequence is the basic lower bound for $\tilde{\sigma}$. If $G$ is a group with $\tilde{\sigma}(G) \geq 3$, then

$$
\tilde{\sigma}(G) \geq 2+|G| / 84 \text {. }
$$

This bound is attained for infinitely many values of $\tilde{\sigma}[\mathbf{1 4}, \mathrm{p} .57]$. In fact, there are infinite families of simple groups that act as groups of $84(p-2)$ automorphisms. Groups of this type include infinitely many of the projective special linear groups 
$\operatorname{PSL}(2, q)[17$, p. 150] as well as the large alternating groups [4]. Here also see $[12, \S 10]$.

An easy, but notable, consequence of (5.2) is that for each integer $\tilde{\sigma} \geq 3$, the number of groups with crosscap number $\tilde{\sigma}$ is finite.

The upper bound $84(p-2)$ can be improved by assuming that $G$ is of a special type; that is, supersolvable, nilpotent, prime order, cyclic, etc. Here, see [3] and [7]. Each improved bound also gives a corresponding lower bound on the crosscap number of the group. For instance, we have the following result.

Proposition B [3]. Let $G$ be a finite nilpotent group with $\tilde{\sigma}(G) \geq 3$. Then $\tilde{\sigma}(G) \geq 2+|G| / 8$.

An extended triangle group is generated by reflections, of course. By Lemma 1, a large group of automorphisms of a non-orientable Klein surface must be generated by involutions. Thus we obtain a lower bound for the crosscap number of a group that is not generated by involutions.

Proposition 1. Let $G$ be a finite group with $\tilde{\sigma}(G) \geq 3$. If $G$ is not generated by involutions, then

$$
\tilde{\sigma}(G) \geq 2+|G| / 24
$$

Proof. Let $G$ act on a non-orientable surface with crosscap number $\tilde{\sigma}=\tilde{\sigma}(G)$. Then, by Lemma $1,|G| \leq 24(\tilde{\sigma}-2)$.

The following example shows that this bound is attained.

ExAmPLE. Let $G=Z_{3} \times \operatorname{PSL}(2,7)$, a group of order $504 ; G$ is obviously not generated by involutions. Let $W$ be a generator for $Z_{3}$ and let $\operatorname{PSL}(2,7) \cong(2,3,7 ; 4)$ have the presentation

$$
R^{2}=S^{3}=(R S)^{7}=[R, S]^{4}=1 .
$$

See [5, p. 96]. Then $G$ has presentation

$$
W^{3}=R^{2}=S^{3}=(R S)^{7}=[R, S]^{4}=[W, R]=[W, S]=1 .
$$

Let $X=W S$. Then $o(X)=3$, and $G=<R, X>$, since $(R X)^{7}=W$. Let $D=[R, X]=[R, S]$, so that $o(D)=4$. Now let $\Delta=\Delta(3,4)$ have presentation $(5.1)$. There is a homomorphism $\phi: \Delta \rightarrow G$ onto $G$ defined by $\phi(c)=R$ and $\phi(x)=X^{-1}$, and $L=$ kernel $\phi$ contains no elements of finite order. It is easy to see that the subgroup $H=<X, D>=<X, R X R>$ is normal in $G$. Then $G / H$ is trivial and $H=G$. Thus $G$ is generated by $X$ and $D$, images of the orientation-preserving elements $x^{-1}$ and $c x c x^{-1}$. Thus $\phi\left(\Delta^{+}\right)=G$ and $L$ is a non-orientable surface group. The group $G$ acts on the surface $U / L$, a non-orientable surface with $c=23$ crosscaps, and $|G|=24(c-2)$.

There is an important connection here with the theory of regular maps on surfaces. For the basic definitions of regular maps, see [5, pp. 20, 101-103]. A map is said to be of type $\{n, k\}$ if it is composed of $n$-gons, $k$ meeting at each vertex. Let $M$ 
be a regular map of type $\{n, k\}$. First assume that $M$ is on an orientable surface. The group of orientation-preserving automorphisms of $M$ is a quotient of $\Gamma(2, n, k)$ with kernel a Fuchsian surface group. If $M$ is reflexible, then the full automorphism group of $M$ is a quotient of $\Gamma[2, n, k]$, with the "rotation" subgroup a quotient of $\Gamma(2, n, k)$. Hence large groups of orientation-preserving automorphisms of Riemann surfaces are map groups. For more on this correspondence, see [16, §8].

Now assume that $M$ is on a non-orientable surface; any regular map on a nonorientable surface is reflexible [5, p. 102]. Then the automorphism group $G$ of $M$ is a quotient of an extended triangle group $\Gamma[2, n, k]$ such that the kernel is a nonorientable surface group. Further there is a reflexible map $N$ on the complex double $X_{c}$ of $X$ which is a double cover of $M$. The full group of the map $N$ is isomorphic to $Z_{2} \times G$. Here, see $[\mathbf{1 7}, \S 4]$ and $[\mathbf{5}, \S 8.1]$. Thus we have the following result.

Lemma 2. Let $G$ be a group of automorphisms of a non-orientable Klein surface $X$ with crosscap number $p \geq 3$. If $G$ is a quotient of a triangle group $\Gamma[2, n, k]$ (with kernel a surface group), then there is a regular map of type $\{n, k\}$ on the topological surface $X$. Further the automorphism group of $X$ is isomorphic to the automorphism group of the map. Conversely, if $G$ is the automorphism group of a regular map of type $\{n, k\}$ on a non-orientable surface $S$, then $G$ is a quotient of a triangle group $\Gamma[2, n, k]$ and $G$ acts as a group of automorphisms of a non-orientable Klein surface homeomorphic to $S$.

For the corresponding result about Riemann surfaces, see [16, p. 28]. Again, for non-orientable surfaces, large groups of automorphisms are map groups.

COROLlary 2. Let $G$ be a group of automorphisms of a non-orientable Klein surface $X$ with crosscap number $p \geq 3$. If $|G|>24(p-2)$, then $G$ is the group of a regular map on a non-orientable surface with p crosscaps.

6. Applications. Here we determine formulas for the crosscap number for three infinite families of groups. We start with a family for which the symmetric genus is known and (4.2) provides the lower bound.

The structure of finite abelian groups is well understood. A finite abelian group $A$ of rank $r$ has a unique canonical form

$$
A=Z_{m_{1}} \times Z_{m_{2}} \times \ldots \times Z_{m_{r}}
$$

such that $m_{i}$ divides $m_{i+1}$ for $i=1, \ldots, r-1$ and $m_{1}>1$ [8, p. 387]. This canonical form is quite useful for studying genus parameters; see [9], for instance.

We consider the abelian groups with most of the factors in the canonical form isomorphic to $Z_{2}$.

THEOREM 2. Let the abelian group $A$ have the canonical form $\left(Z_{2}\right)^{a} \times Z_{m_{1}} \times \ldots \times Z_{m_{b}}$, where $a>b \geq 0$ and $m_{1}>2$. Then

$$
\tilde{\sigma}(A)=2+|A|(3 b+a-3) / 4 \text {. }
$$

Proof. The formula holds for the abelian groups with $\tilde{\sigma} \leq 2$. Assume $\tilde{\sigma}(A) \geq 3$, so that either $b>0$ or $a \geq 4$. Let $\Delta$ be an NEC group with signature 


$$
\left(0 ;+;[] ;\left\{()^{b},\left(2^{a-b+1}\right)\right\}\right) \text {. }
$$

We calculate

$$
\mu(\Delta) / 2 \pi=(3 b+a-3) / 4 .
$$

Write $t=a-b+1$. Then $\Delta$ has a presentation with generators $c_{1}, \ldots, c_{b}, d_{0}, \ldots, d_{t}$, $e_{1}, \ldots, e_{b}, f$ (the $d_{i}$ 's and $f$ correspond to the last period cycle) and relations

$$
\begin{gathered}
\left(c_{i}\right)^{2}=\left(d_{j}\right)^{2}=\left[e_{i}, c_{i}\right]=e_{1} e_{2} \ldots e_{b} f=1, \\
\left(d_{0} d_{1}\right)^{2}=\left(d_{1} d_{2}\right)^{2}=\ldots=\left(d_{t-1} d_{t}\right)^{2}=1, f d_{0} f^{-1}=d_{t} .
\end{gathered}
$$

Now let $V_{1}, \ldots, V_{a}$ be a set of generators for $\left(Z_{2}\right)^{a}$, and let $W_{j}$ be a generator for the factor $Z_{m_{j}}$. Then there is a homomorphism $\phi: \Delta \rightarrow A$ onto $A$ defined by

$$
\begin{gathered}
\phi\left(c_{i}\right)=V_{i} \quad(i=1, \ldots, b), \\
\phi\left(d_{j}\right)=V_{b+j} \quad(j=1, \ldots, a-b), \\
\phi\left(e_{i}\right)=W_{i} \quad(i=1, \ldots, b), \\
\phi\left(d_{0}\right)=\phi\left(d_{t}\right)=V_{b+1} V_{b+2}, \phi(f)=\left(W_{1} W_{2} \ldots W_{b}\right)^{-1} .
\end{gathered}
$$

The homomorphism $\phi$ is clearly onto. By considering the images of (orientationpreserving) products of two reflections (along with the images of the connecting generators $\left.e_{1}, \ldots, e_{b}\right)$, it is not hard to see that $\phi\left(\Delta^{+}\right)=A$. Further, $L=$ kernel $\phi$ contains no elements of finite order. Hence $L$ is a non-orientable surface group, and $A$ acts on the non-orientable surface $Y=U / L$. If $Y$ has $p$ crosscaps, then from (2.4) $p=2+|A|(3 b+a-3) / 4$. Hence, $\tilde{\sigma}(A) \leq 2+|A|(3 b+a-3) / 4$.

On the other hand, $\tilde{\sigma}(A) \geq \sigma\left(Z_{2} \times A\right)+1$, by (4.2). Clearly, the canonical form of $Z_{2} \times A$ is $\left(Z_{2}\right)^{a+1} \times Z_{m_{1}} \times \ldots \times Z_{m_{b}}$, where the number of $Z_{2}$ factors $a+1 \geq b+2$. Using the formula for the symmetric genus from [9, Theorem 5.3, p. 420], we have

$$
\sigma\left(Z_{2} \times A\right)=1+\left|Z_{2} \times A\right|[(a+1)+3 b-4] / 8=1+|A|[a+3 b-3] / 4 .
$$

This gives the lower bound, and we have the formula for the crosscap number.

As a special case, we obtain the formula for the symmetric crosscap number of an elementary abelian 2-group.

COROLlaRy 3. $\tilde{\sigma}\left(Z_{2}\right)^{a}=2+2^{a-2}(a-3)$.

For $n \geq 2$, let $D C_{n}$ be the dicyclic group or order $4 n[5, \mathrm{p} .7]$ with generators $X$, $Y$ and defining relations

$$
X^{2 n}=1, X^{n}=Y^{2}, Y^{-1} X Y=X^{-1} .
$$

Each element outside the cyclic subgroup $\langle X>$ has order 4 and hence there must be at least one element of order 4 in any generating set for $D C_{n}$. There is a unique element $\left(X^{n}\right)$ of order 2 in the group, and clearly $X^{n}$ is in the center. It is also not 
hard to see that $X^{n}$ is in the Frattini subgroup and is a non-generator. We know that $\sigma^{0}\left(D C_{n}\right)=n$, if $n$ is even, and $\sigma^{0}\left(D C_{n}\right)=n-1$, if $n$ is odd, by [11, Theorem 1]. By (4.1) we have $\tilde{\sigma}\left(D C_{n}\right) \geq 3$, for all $n$.

TheOREM 3. If $n \neq 3$, then $\tilde{\sigma}\left(D C_{n}\right)=2 n+2$. Furthermore $\tilde{\sigma}\left(D C_{3}\right)=7$.

Proof. Write $G=D C_{n}$, and let $G$ have presentation (6.1). Let $G$ act on the nonorientable Klein surface $W$ of topological genus $\tilde{\sigma}=\tilde{\sigma}(G) \geq 3$. Represent $W$ as $U / K$, where $K$ is a non-orientable surface group, to obtain a proper NEC group $\Gamma$ with signature (2.1) and a homomorphism $\phi: \Gamma \rightarrow G$ onto $G$ such that kernel $\phi=K$. Write $A=\mu(\Gamma) / 2 \pi$. Then, by (2.4), $\tilde{\sigma}=2+4 n A$. We obtain a lower bound for $A$. Since $\Gamma$ is a proper NEC group, either $k \neq 0$ (and the surface $U / \Gamma$ is bordered) or the minus sign is present in the signature (and $U / \Gamma$ is non-orientable) or both.

I. Suppose first that $k=0$, so that $U / \Gamma$ must be non-orientable. If $p \geq 3$, then $A \geq 1$. If $p=2$, then $r \geq 1$ (since $A>0$ ) and easily $A \geq 1 / 2$. Assume $p=1$. Then clearly $r \geq 2$. If $r \geq 3$, it follows that $A \geq-1+3(1 / 2)=1 / 2$. Assume $r=2$ so that $\Gamma$ is an NEC group with signature $\left(1 ;-;\left[\lambda_{1}, \lambda_{2}\right] ;\{\}\right)$, where $\lambda_{1} \leq \lambda_{2}$. Then $\Gamma$ has presentation

$$
s^{\lambda_{1}}=t^{\lambda_{2}}=s t a^{2}=1
$$

The quotient group $G \cong \Gamma / K$ is generated by the two elements $\phi(s)$ and $\phi(a)$ (also by $\phi(t)$ and $\phi(a))$. Since the unique involution of $G$ is a non-generator, immediately we have $\lambda_{2} \geq \lambda_{1} \geq 3$.

Suppose that $G=D C_{n}$ has elements of order 3 . Then 3 divides $n$, of course. We write $n=3 \ell$. But the two elements of $G$ of order 3 are contained in the normal subgroup $N=<X^{2 \ell}>$, and it is not hard to see that the quotient group $G / N$ is the dicyclic group $D C_{\ell}$ if $\ell>1$. Hence if $\ell>1$, an element of order 3 cannot be part of a two-element generating set for $D C_{n}$. Also, $D C_{3}$ is not generated by two elements of order 3 . For the group $D C_{3}$, if $\lambda_{1}=3$, then $\lambda_{2} \geq 4$ and $A \geq-1+(2 / 3)+3 / 4$; thus $\tilde{\sigma}\left(D C_{3}\right) \geq 7$.

Assume that $n \neq 3$. Whether $D C_{n}$ has elements of order 3 or not, we must have $\lambda_{1} \geq 4, \lambda_{2} \geq 4$. Then we obtain $A \geq-1+2(3 / 4)=1 / 2$. Hence $\tilde{\sigma} \geq 2 n+2$, in general.

II. Suppose next that $k \neq 0$ so that the quotient space $U / \Gamma$ is a bordered surface. Let $\gamma$ denote the algebraic genus of $U / \Gamma$. We simplify the presentation for $\Gamma$ as in [10, Lemma 1, p. 118]. In this simplified presentation there must be at least two elements with order larger than two, since $\Gamma / K \cong D C_{n}$. It follows that

$$
\gamma+r \geq 2
$$

If $\gamma \geq 2$, it is easily seen that $A \geq 1$. Suppose that $\gamma=1$. Then we have $r \geq 1$ with at least one ordinary period larger than two, and $A \geq 2 / 3$.

Now assume $\gamma=0$, so that $U / \Gamma$ is the disc $D$. Then $r \geq 2$, with at least two of the ordinary periods greater than two. If $r \geq 3$, then it is easy to see that $A \geq-1+2(2 / 3)+1 / 2=5 / 6$. Suppose that $r=2$. Then $\Gamma$ has signature $(0 ;+$; $\left.\left[\lambda_{1}, \lambda_{2}\right] ;\{C\}\right)$, where $C$ is a period cycle and $\lambda_{1} \leq \lambda_{2}$. The group $\Gamma$ is generated by $s, t$, the connecting generator $e$ and some reflections. But ste $=1$, and $e$ is redundant. Further, if $c$ is a reflection, the $\phi(c)$ must be the unique involution of $G$. Thus $G$ is 
generated by the two images $\phi(s)$ and $\phi(t)$ with orders $\lambda_{1}$ and $\lambda_{2}$, respectively. Now, just as before, for $D C_{3}$, if $\lambda_{1}=3, \lambda_{2} \geq 4$ and we have $\tilde{\sigma} \geq 7$. if $n \neq 3$, then we must have $\lambda_{1} \geq 4, \lambda_{2} \geq 4$, and $\tilde{\sigma} \geq 2 n+2$ in general again.

The group $G$ is generated by the two elements $X Y$ and $Y$ of order 4 , and $X^{n}$ is an involution in the center of $G$. Thus the upper bound of Theorem 1 is $\tilde{\sigma} \leq 2+4 n \cdot 1 / 2=2+2 n$, in agreement with the lower bound. Also, the group $D C_{3}$ is generated by $X^{2}$ and $Y$, elements of orders 3 and 4 ; in this case $\tilde{\sigma} \leq 7$, by Theorem 1 .

A hamiltonian group is a non-abelian group in which every subgroup is normal. The finite hamiltonian groups have the form

$$
Q \times A \times B
$$

where $Q \cong D C_{2}$ is the quaternion group, $\mathrm{A}$ is an elementary abelian 2-group and $B$ is an abelian group of odd order [5, p. 8]. We shall consider the hamiltonian groups with no odd order part; the symmetric genus of these groups was determined in $[\mathbf{1 0}$, Theorem 3].

THEOREM 4. Let $G=\left(Z_{2}\right)^{\mathrm{a}} \times Q$. Then

$$
\tilde{\sigma}(G)= \begin{cases}2+2^{a+1}(a+2), & \text { if } a=1 \text { or } a=2 \\ 2+2^{a+1}(a+3), & \text { if } a \geq 3 .\end{cases}
$$

Proof. First suppose that $a \geq 3$. Let $\Gamma$ be an NEC group with signature

$$
\left(0 ;+;[4,4] ;\left\{\left(2^{a+1}\right)\right\}\right. \text {. }
$$

We calculate $\mu(\Gamma) / 2 \pi=(a+3) / 4$. The group $\Gamma$ has a presentation with generators $x, y, c_{0}, \ldots, c_{a+1}, e$ and relations

$$
\begin{gathered}
x^{4}=y^{4}=\left(c_{i}\right)^{2}=x y e=1, \\
\left(c_{0} c_{1}\right)^{2}=\left(c_{1} c_{2}\right)^{2}=\ldots=\left(c_{a} c_{a+1}\right)^{2}=1, \quad e c_{0} e^{-1}=c_{a+1} .
\end{gathered}
$$

Let $V_{1}, \ldots, V_{a}$ be a set of generators for $\left(Z_{2}\right)^{a}$, and let $X$ and $Y$ be generators for $Q$ satisfying (6.1). Then there is a homomorphism $\phi: \Gamma \rightarrow G$ onto $G$ defined by

$$
\begin{gathered}
\phi(x)=X, \quad \phi(y)=Y, \\
\phi\left(c_{i}\right)=V_{i} \quad(i=1, \ldots, a), \\
\phi\left(c_{0}\right)=\phi\left(c_{a+1}\right)=V_{1} V_{2}, \quad \phi(e)=(X Y)^{-1} .
\end{gathered}
$$

The homomorphism $\phi$ is clearly onto. By considering the images of products of two reflections (together with the images of $x$ and $y$ ), it is not hard to see that $\phi\left(\Gamma^{+}\right)=G$. Further, $K=$ kernel $\phi$ has no elements of finite order. Hence $K$ is a non-orientable surface group, and $G$ acts on the surface $Y=U / K$. If $p$ is the crosscap number of $Y$, then from (2.4) we find that $p=2+2^{a+1}(a+3)$. Hence $\tilde{\sigma}(G) \leq 2+2^{a+1}(a+3)$ in the general case.

If $a<3$, then it is possible to improve the upper bound slightly. If $a=1$, then $G$ is a quotient of an NEC group with signature $\left(0 ;+;[4] ;\left\{()^{2}\right\}\right)$ such that the kernel is a non-orientable surface group. If $a=2$, then use an NEC group with signature $\left(0 ;+;[] ;\left\{()^{3}\right\}\right)$. In these two cases, $\tilde{\sigma}(G) \leq 2+2^{a+1}(a+2)$. 
Again, $\tilde{\sigma}(G) \geq \sigma\left(Z_{2} \times G\right)+1$, by (4.2) and clearly $Z_{2} \times G \cong\left(Z_{2}\right)^{a+1} \times Q$. Using the formula for the symmetric genus from [10, p. 122], we have $\sigma\left(Z_{2} \times G\right)=$ $1+2^{a+1}(a+3)$ if $a \geq 3$ and $\sigma\left(Z_{2} \times G\right)=1+2^{a+1}(a+2)$ if $a=1$ or $a=2$. This gives the lower bound, and we have the formula for the crosscap number.

7. Small groups. Theorem 3 completes the calculation of the symmetric crosscap number of each group of order less than 16 . The cyclic groups have symmetric crosscap number 1 , and the abelian groups $Z_{2} \times Z_{n}$ of rank two have $\tilde{\sigma}=2$. The only other abelian groups in the range are $\left(Z_{2}\right)^{3}$ and $Z_{3} \times Z_{3}$; we know $\tilde{\sigma}\left(Z_{3} \times Z_{3}\right)=5[\mathbf{1 2}, \S 9]$.

The dihedral groups $D_{n}$ have symmetric crosscap number 1 . If $n$ is even, then $\tilde{\sigma}\left(Z_{2} \times D_{n}\right)=2$; here, of course, $\left(Z_{2}\right)^{3} \cong Z_{2} \times D_{2}$. Theorem 3 deals with the dicyclic groups. The only other non-abelian group in the range is $A_{4}$, which has symmetric crosscap number 1 . The following table gives $\tilde{\sigma}(G)$ as well as $\sigma^{0}(G)$ and $\sigma(G)$, for each group $G$ with $|G|<16$ and $\tilde{\sigma}(G)>2$.

\begin{tabular}{ccccc}
\multicolumn{5}{c}{ Groups of small order with $\tilde{\sigma}>2$} \\
order & group $G$ & $\tilde{\sigma}(G)$ & $\sigma^{0}(G)$ & $\sigma(G)$ \\
8 & $Q$ & 6 & 2 & 1 \\
9 & $Z_{3} \times Z_{3}$ & 5 & 1 & 1 \\
12 & $D C_{3}$ & 7 & 2 & 1
\end{tabular}

8. Crosscap number 3. Finally we use regular maps to classify the groups with crosscap number 3. Suppose that $G$ is a finite group with $\tilde{\sigma}(G)=3$. The group $G$ must act on a non-orientable Klein surface $X$ with 3 crosscaps and Euler characteristic -1 . The complex double $X_{c}$ is a Riemann surface with Euler characteristic $\chi\left(X_{c}\right)=-2$ and genus 2 .

First we apply Lemma 1 and the correspondence between large groups and regular maps. If $|G|>24$, then $G$ is a group of a regular map on a surface with 3 crosscaps. If $24 \geq|G|>12$, then $G$ is a group of a regular map unless $G$ is a quotient of one of the exceptional groups in cases (1), (2), (4), (5) or (6); in these cases, $G$ has order 24,20 or 15 .

Thus, if $G$ is a group with crosscap number 3 , then either $G$ is the group of a regular map on a non-orientable surface with 3 crosscaps, $G$ is a small group of order at most 12, or $G$ is a quotient of one of the five exceptional NEC groups.

However, there are no regular maps at all on a surface with 3 crosscaps [5, p. 116], and none of the small groups have $\tilde{\sigma}=3$. We need to consider the exceptional groups.

The only group of order 15 is cyclic, of course, and has no involutions. Hence $Z_{15}$ is clearly not a quotient of either $\Gamma[3,3,5]$ or $\Delta(3,5)$.

Suppose that $|G|=20$ and $G$ were a quotient of $\Delta=\Delta(5,2)$ by a non-orientable surface group $K$. Then $\Delta^{+}=\Gamma(2,5,5)$ and $\Delta^{+} / K^{+} \cong \Delta / K \cong G$ is the rotation group of a regular map of type $\{5,5\}$ on the complex double $X_{c}=U / K^{+}$, a surface of genus 2 . The regular maps on an orientable surface of genus 2 have been classified [5, p. 140], and none of these maps are of type $\{5,5\}$. Thus this exceptional case does not occur.

Finally suppose that $|G|=24$ and $G$ were a quotient of the NEC group $\Lambda$ by a non-orientable surface group $K$, where $\Lambda$ is either $\Gamma[3,3,4]$ or $\Delta(3,4)$. In either case $\Lambda^{+}=\Gamma(3,3,4)$ and $\Lambda^{+} / K^{+} \cong G$. It follows that $G \cong \operatorname{SL}(2,3)[\mathbf{1 0}$, pp. 125,127]; $G$ is also called the binary tetrahedral group $<2,3,3>$. See [5, pp. 68,69]. But the binary tetrahedral group has a unique involution $T$ such that the center $Z=<T>$ and 
$G / Z \cong A_{4}$, the ordinary tetrahedral group. Now $G$ is obviously not a quotient of either $\Gamma[3,3,4]$ or $\Delta(3,4)$ by a non-orientable surface group, and these exceptional cases do not occur either.

In summary we have the following result.

\section{THEOREM 5. There are no groups with symmetric crosscap number 3.}

This affirms a conjecture of Tucker [19, p. 1118]. It also raises the interesting question of whether there are other integers for which there are no groups with that symmetric crosscap number. We do not yet know enough about this parameter to make a confident conjecture. However, it has recently been established that if $n$ is a non-negative integer, then there is at least one group of strong symmetric genus $n$ [13, Theorem 1]. The related problem about the symmetric genus remains unsolved; see $[11, \S 9]$.

Finally, we would like to thank the referee for several helpful suggestions.

\section{REFERENCES}

1. N. L. Alling and N. Greenleaf, Foundations of the theory of Klein surfaces, Lecture Notes in Mathematics No. 219 (Springer-Verlag, 1971).

2. E. Bujalance, J. J. Etayo, J. M. Gamboa, and G. Gromadzki, Automorphism groups of compact bordered Klein surfaces, Lecture Notes in Mathematics No. 1439 (Springer-Verlag, 1990).

3. E. Bujalance and G. Gromadzki, On nilpotent groups of automorphisms of Klein surfaces, Proc. Amer. Math. Soc. 108 (1990), 749-759.

4. M. D. E. Conder, Generators for alternating and symmetric groups, J. London Math. Soc. (2) 22 (1980), 75-86.

5. H. S. M. Coxeter and W. O. J. Moser, Generators and relations for discrete groups, Fourth Edition (Springer-Verlag, 1957).

6. L. Greenberg, Maximal Fuchsian groups, Bull. Amer. Math. Soc. 69 (1963), 569-573.

7. G. Gromadski, Supersoluble groups of automorphisms of nonorientable Riemann surfaces, Bull. London Math. Soc. 22 (1990), 561-568.

8. S. MacLane and G. Birkoff, Algebra (Macmillan, New York, 1979).

9. C. L. May and J. Zimmerman, The symmetric genus of finite abelian groups, Illinois J. Math. 37 (1993), 400-423.

10. C. L. May and J. Zimmerman, Groups of small symmetric genus, Glasgow Math. J. 37 (1995), 115-129.

11. C. L. May and J. Zimmerman, Groups of small strong symmetric genus, J. Group Theory 3 (2000), 233-245.

12. C. L. May and J. Zimmerman, The group of symmetric Euler characteristic-3, to appear in Houston J. Math. (2002).

13. C. L. May and J. Zimmerman, There is a group of every strong symmetric genus, to appear.

14. D. Singerman, Automorphisms of compact non-orientable Riemann surfaces, Glasgow Math. J. 12 (1971), 50-59.

15. D. Singerman, On the structure of non-Euclidean crystallographic groups, Math. Proc. Cambridge Philos. Soc. 76 (1974), 233-240.

16. D. Singerman, Symmetries of Riemann surfaces with large automorphism group, Math. Ann. 210 (1974), 17-32.

17. D. Singerman, PSL $(2, q)$ as an image of the extended modular group with applications to group actions on surfaces, Proc. Edinburgh Math. Soc. (2) 30 (1987), 143-151.

18. T. W. Tucker, Finite groups acting on surfaces and the genus of a group, J. Combin. Theory Ser. B 34 (1983), 82-98.

19. T. W. Tucker, Symmetric embeddings of Cayley graphs in non-orientable surfaces, in Graph theory, combinatories and applications, edited by Y. Alavi, G. Chartrand, O. R. Ollerman and A. J. Schwenk (1991), 1105-1120. 